\title{
INFORMATIONS SFG
}

\section{Réunions scientifiques SFG}

\section{- Génétique et société}

14-15 février 1991

Colloque suivi de la réunion de l'assemblée générale de la Société française de génétique

Lieu : Institut Pasteur, 28, rue du Docteur-Roux, 75015 Paris. La génétique inquiète et attire de plus en plus nos concitoyens, comment pouvons-nous répondre à cette demande et prendre en compte et/ou apaiser ces inquiétudes?

Programme prévisionnel :

Jeudi 14 février : 1 "La génétique et l'homme " : 1.1 "Espoirs et dangers" : les manipulations génétiques, les bénéfices escomptés, génétique humaine et éthique, la prédiction des risques et son utilisation sociale. Discussion générale - Table ronde - 1.2 "Avancées scientifiques et techniques".

La journée se terminera par l'assemblée générale de la Société française de génétique.

Vendredi 15 février : 2 "Les autres espèces": 2.1 "les microorganismes": les virus, les bactéries, les levures, 2.2 "les végétaux": le point de vue du secteur privé, la législation anglaise, 2.3 "Les animaux": les espèces domestiques, les espèces de laboratoire. - Discussion et conclusions - L'après-midi sera consacré à une table ronde, réunion du Cnrs.

Organisation: Antoine Danchin, Régulation de l'expression génétique, Institut Pasteur, 25, rue du Docteur-Roux, 75015 Paris, et Pierre-Henri Gouyon, Évolution et systématique des végétaux - bâtiment 362, université Paris-Sud, 91405 Orsay Cedex.

- Levure : modèle et outil

\section{8-29 mars 1991}

La Société française de génétique organise un colloque : "La levure : modèle et outil" les 28 et 29 mars 1991 à l'Institut Pasteur à Paris. Ce colloque s'adresse aussi bien aux "levuristes " qu'aux "non-levuristes " désireux d'approfondir leurs connaissances. Il comportera des communications courtes (15 minutes) et des affiches.

Les thèmes proposés sont les suivants : facteurs de transcription ; maturation des ARN ; interaction ARN-protéine ; recombinaisons génétiques; maintien et fluidité du génome ; organelles et métabolisme respiratoire ; transport membranaire et sécrétion ; signalisation intracellulaire et cycle ; ingénieries génétiques et moléculaires; projets Génome.

S'adresser au secrétariat de la SFG, Unité d'immunogénétique humaine, Institut Pasteur, 25, rue du Docteur-Roux, F-75724 Paris Cedex 15, Fax: (33) 1.45.68.86.39.

\section{- Cartographie comparée des mammifères}

30-31 mai 1991

Placée sous le patronage de la Société française de génétique, cette réunion aura lieu au Centre Inra de Toulouse-Auzeville. Objectifs : Stratégies d'élaboration des cartes géniques chez les mammifères et leurs applications aux espèces domestiques.

Thèmes principaux : - Application des connaissances acquises chez l'homme et la souris pour l'avancement de la cartographie génique des espèces domestiques. - Mise au point sur les techniques actuelles de la cartographie et leur évolution (hybridation in situ, tri de chromosomes, électrophorèse en champ pulsé, analyse de ségrégation génique sur gamètes). -
QTL, hérédité des caractères complexes. Cette réunion comprendra des conférences d'environ 30 minutes et des communications sous forme de posters.

Comité d'organisation : J. Gellin, F. Grosclaude, M. Dalens, G. Echard, F. Gasser, M. Yerle.

Coordinateur : Dr J. Gellin, Inra, Laboratoire de génétique cellulaire, BP 27, 31326 Castenet-Tolosan -Tél. : (16) 61.28.51.15 - Fax : (16) 61.28.52.80.

\section{- Sexe et évolution}

\section{Mai 1991}

Avec la participation de la Société française de biologie du développement

Lieu : Institut Pasteur, 28, rue du Docteur-Roux, 75724 Paris Cedex 15.

Contacter : Marc Fellous, Immunogénétique humaine, Institut Pasteur, 25, rue du Docteur-Roux, 75724 Paris Cedex 15, et Michel Dauça, Laboratoire de biologie cellulaire du développement, Faculté des sciences, BP 239, 54506 Vandœuvre-lèsNancy Cedex.

\section{Autres colloques, réunions et congrès}

\section{- Calendar of Genome Events}

January 1991, 8-11 : Biotechnology Computing Minitrack at the Hawaii International Conference on System Sciences-24; Kailua-Kona, HI (L. Hunter, (301) 496-9300, Fax : (301) 496-0673).

January 1991, 27-February 1: Bio/Technology Magazine Winter Symposium-Advances in Gene Technology: The Molecular Biology of Human Genetic Disease ; Miami Beach FL (The Miami Bio/Technology Winter Symposia, (800) 624-4363, Fax : (305) 324-5665.

February 1991, 24-28 : "Special Mini-Track ou Artificial Intelligence Application to Molecular Biology " at the Seventh IEEE Conference on Artificial Intelligence Applications, Miami Beach (David Searls, (215) 648-2146.

March 1991, 19-21 : Sessions on "Development and Application of Electrophoresis Techniques in Molecular Biology " at the International Electrophoresis Society Meeting, Washington DC ; J. Cunningham, (301) 898-3772, Fax : (301) 898-5596. July 1991, 22-26 : 13th IMACS World Congress on Computation and Applied Mathematics with sessions on High Performance Computing in Biology and Medicine ; Dublin, Ireland (M. Witten, USA; (512) 471-2449.

August 1991, 18-22 : 11th International Workshop on Human Gene Mapping (HGM 11) ; London UK (M. Probert), (44-71) 2693052 - Fax Int. (44-71) 430-1787.

August 1991, 25-31 : XXII International Conference on Animal Genetics, East Lansing MI (R. Bull), (517) 355-4616, Fax : (517) 353-5436.

October 1991, 6-11 : 8th International Congress of Human Genetics, Washington DC (ICHG), (301) 571-1825, Fax : (301) 530-7079). 


\section{INFORMATIONS SFG}

- 7th Tenovus - Scotland Symposium " Eukaryotic genes and their expression"

University of Glasgow, 20-22 mars 1991

Date limite pour inscription et abstracts : 18 janvier 1991. Contacter Dr R.L.P. Adams, Department of Biochimistry, University of Glasgow, Glasgow G12 8QQ UK.

- 8th International Congress of Human Genetics

Washington DC, 6-11 octobre 1991

Contacter the 8th International Congress of Human Genetics, Administrative Office, 9650 Rockville Pike - Bethesda Maryland 20814, USA.

- Spring Meeting 1991 "Genes and proteins " University of York, 9-11 avril 1991

Contacter Dr Susan M. Bougourd, Department of Biology, University of York, York Y01 5DD.

- Meeting of SGM Genetics and Molecular Biology Group " Genetics of virulence in Gram-positive bacteria "

9-12 avril 1991

Contacter Pr. P.A. Williams, School of Biological Sciences, University College of North Wales, Bangor, Gwynedd LL57 2UW (tel. : 0248 351151).

- Meeting of British Ecological Society "Genes in ecology " University of East Anglia, Norwich

3-5 avril 1991

Contacter Pr. R.J. Berry, Departement of Biology, University

College London, Gower Street, London WC1E 6BT.

\section{Informations diverses}

\section{- Concours INRA}

1) Concours de Chargé de recherche (Concours $C R$ ) : dépôt des candidatures mars 1991.

2) Concours de Directeur de recherche de $2^{\mathbf{e}}$ classe (Concours $D R 2)$ : dépôt des candidatures février 1991.

3) Concours d'Attaché scientifique contractuel (Concours ASC) : dépôt des candidatures mai 1991.

Demandes de dossier et informations auprès du Service du personnel, INRA, Division des chercheurs, 147, rue de l'Université, 75341 Paris Cedex 07 - Dans tous les cas, obtenir confirmation des dates auprès du Service du personnel, Division des chercheurs - Tél. : (1) 42.75.90.00.

- Ministère de l'Éducation nationale, de la Jeunesse et des Sports

Organigramme de la Direction de la recherche et des études doctorales (DRED), 61-65, rue Dutot, 75732 Paris Cedex 15. Tél. : (1) 40.65.65.40.

Composition de la 5e Direction scientifique "Sciences de la Vie et de la Santé " - Tél. : (1) 40.65.61.65.

Directeur scientifique : M. Philippe Vigier - Consultants : MM. Pierre Bourlioux, Alain Fischer, Jean-Claude Patte, Georges Périquet, Jean-Paul Rousseau, Régis Mache.

Coordinatrice scientifique : Mme Danièle Hoursiangou.

\section{SOCIÉTÉ FRANC̣AISE DE GÉNÉTIQUE}

\section{DEMANDE D'ADHÉSION}

Cette demande est à adresser au secrétaire général, R. Motta, laboratoire d'immunogénétique, centre Marcel-Delépine, Cnrs, 1, rue Haute, 45071 Orléans Cedex 2. (Conformément aux statuts, elle sera soumise à I'approbation du bureau dans sa prochaine séance.)

NOM (Madame, Mademoiselle, Monsieur) :

PRÉNOM

DATE DE NAISSANCE :

NATIONALITÉ :

FONCTION ET GRADE :

ORGANISME DE RATTACHEMENT :

TITRES ET DIPLÔMES

ADRESSE PERSONNELLE* (adresse, téléphone) :

ADRESSE PROFESSIONNELLE* (centre ou université, laboratoire. Adresse, téléphone) :

DATE :

SIGNATURE :

Montant de la cotisation 1990 : $200 \mathrm{~F}$ (100 F pour les étudiants - joindre photocopie de la carte d'étudiant) par chèque libellé à l'ordre de la SOCIÉTÉ FRANÇAISE DE GÉNÉTIQUE.

* Marquer d'une croix celle où doit être adressé le courrier. 